\title{
Femtosecond Filamentation in Temperature Controlled Noble Gas
}

\author{
Zhenming Song ${ }^{2}$, Yun Wei ${ }^{1}$, Shiying Cao ${ }^{1}$, Weipeng Kong2, \\ Dongqing Pang2, Ruxin $\mathrm{Li}^{3}$, Qingyue Wang 2 and Zhigang Zhang ${ }^{1}$ \\ ${ }^{1}$ Institute of Quantum Electronics, State Key Laboratory of Advanced Optical \\ Communication Systems and Networks, School of Electronics Engineering and Computer \\ Science, University of Beijing, Beijing 100871 \\ ${ }^{2}$ Ultrafast Laser Laboratory, School of Precision Instrument and Optoelectronics \\ Engineering, Key Laboratory of Optoelectronic Information Technical Science, EMC, \\ University of Tianjin, Tianjin 300072 \\ ${ }^{3}$ State Key Laboratory of High Field Laser Physics, Shanghai Institute of Optics and Fine \\ Mechanics, Chinese Academy of sciences, P.O. Box 800-211 Shanghai 201800
}

China

\section{Introduction}

Intense few to monocycle pulses are the $\mathrm{mJ}$ or sub-mJ ultrashort pulses which have one to two optical periods ( $800 \mathrm{~nm}$ corresponding to $2.67 \mathrm{fs})$. They play more and more important roles in today's scientific and technological research fields, such as time-resolved measurements of electron dynamics in atoms and molecules, high-order harmonics and isolate attosecond pulse generation. To date, the major way of achieving intense few to monocycle pulses is to compress the spectra broadened through hollow fiber filled with noble gases or filamentation.

Combined with the technique of noble gas-filled hollow fiber and feedback phase compression, Yamashita group has achieved the shortest pulse duration $2.6 \mathrm{fs}$ in the visible light range (Yamashita et al., 2006; Yamane et al., 2003; Matsubara et al., 2007). However, due to the damage at the entrance of the fiber, self-focusing and gas breakdown, the pulse energy in a gas-filled hollow fiber is limited to $\mu \mathrm{J}$ level. In addition, the loss in the hollow fiber also contributes to the low efficiency. Spectrum broadening through filamentation was presented as one of the extremely simple technique to generate intense few to monocycle pulses (Hauri et al. 2004; Hauri et al., 2005; Zaïr et al., 2007). In addition, the pulse selfcompression can be realized in the filamentation (Couairon et al., 2006; Couairon et al., 2005). However, the competition of the multi-filament (Shen, 1984; Bergé et al., 2004; Vidal \& Johnston, 1996; Braun et al., 1995; Skupin et al., 2004; Hosseini et al., 2004) when the peak power is several times above the critical power introduces instability between the filaments and restricts the achievement of high quality ultrashort intense pulses. The spectrum broadening through hollow fiber with a gradient pressure seems to be an efficient way to overcome the breakdown at the entrance of the fiber, and preserve the spatial and spectral qualities of the pulses with final energy of multi-mJ (Suda et al., 2005; Sung et al., 2006), but

Source: Advances in Solid-State Lasers: Development and Applications, Book edited by: Mikhail Grishin,

ISBN 978-953-7619-80-0, pp. 630, February 2010, INTECH, Croatia, downloaded from SCIYO.COM 
it is difficult to realize in the filamentation, because differential pumping does not only result in a large consumption of expensive noble gases, but also in a gas flow that disturbs the spatial beam stability and the spectral characteristics.

In this Chapter, we present a novel technique that could have the same effect as gradient pressure scheme to produce controllable nonlinear refraction index: the gradient temperature in a sealed tube filled with noble gas (Song et al., 2008a; Song et al., 2008b; Cao et al., 2009). That is, in practice, to heat the entrance and cool the exit end of the tube, so a temperature gradient can be formed along the tube. The gas pressure in this sealed tube reaches equilibrium but the gas density is gradually increased along the tube. This gradient temperature should have the similar effect as that of gradient pressure, because these two schemes both have increasing gas density distribution along the propagation of the pulse. Therefore, the incident pulse will be allowed to have a higher energy because the lower gas density at the entrance. The spectrum broadening will go on along the tube because the increasing gas density will compensate the decreasing peak power due to loss and dispersion. The ideas of gradient pressure and gradient temperature schemes are shown in Fig. 1. In the gradient pressure case the gas flow has disturbance to the system which will affect the stability and quality of the output beam while in the gradient temperature case the gas reaches equilibrium to avoid the disadvantages of the gradient pressure. Theoretical and experimental results will be shown in section 3 and 4 respectively to illustrate and prove the feasibility and effectiveness of the gradient temperature scheme and a final conclusion will be drawn in section 5 of this Chapter. Before we show the results we shall simply make some efforts on the filamentation and dynamics in filamentation in the section below.

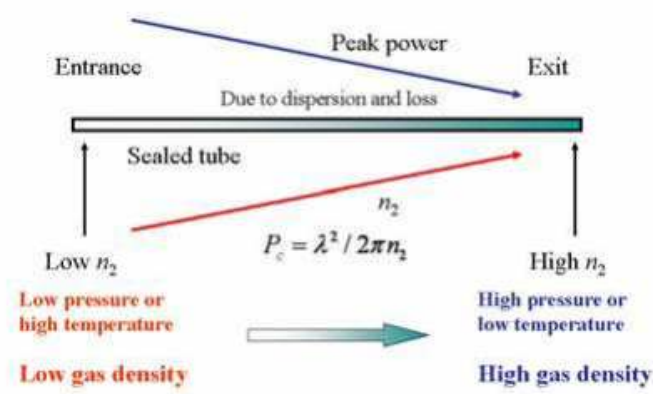

Fig. 1. Illustration of the gradient pressure and gradient temperature technique.

\section{Theoretical model of dynamics in filamentation}

Filamentation is a well-known phenomenon of the propagation of intense laser pulses in any kinds of Kerr media (gases, liquids, and transparent solid materials) under certain conditions. It is the result of the balance between the self-focusing due to the nonlinear refractive index of the medium dependence on the intensity and the defocusing due to the formation of the plasma and beam diffraction when a sufficient intense ultrashort laser 
pulse propagates in the transparent medium. A single filament always occurs when the incident pulse with a peak power between one and a few times $P_{c}$ where $P_{c}$ is the critical power values of self-focusing. For a CW Gaussian beam, the self-focusing critical power is expressed by (Marburger, 1975):

$$
P_{c}=\lambda_{0}^{2} / 2 \pi n_{0} n_{2}
$$

where $\lambda_{0}$ is the wavelength in vacuum, $n_{0}$ and $n_{2}$ are the linear and nonlinear refraction index of the medium. However, when the laser peak power is much higher $r$ than the selffocusing critical power, the incident beam typically breaks into several long and narrow filaments, a phenomenon known as multiple filamentation (MF). Multiple filamentation is an unstable state because there is the interaction among the filaments (Shen, 1984; Bergé et al., 2004; Vidal \& Johnston, 1996; Braun et al., 1995; Skupin et al., 2004; Hosseini et al., 2004), which limits the energy that each filament can carry.

\subsection{Model equations}

In order to describe the dynamic of filamentation, a 3D extended nonlinear Schrödinger equation coupled with the electron density equation are usually employed. The extended nonlinear Schrödinger equation for the electric field envelop $\varepsilon(r, t, z)$ in a reference frame $(t=$ $t_{\text {lab }}-z / v_{g}$ ) moving at the group velocity $v_{g}$ can be written as,

$$
\begin{aligned}
& \partial \varepsilon / \partial z=i\left(\partial^{2} \varepsilon / \partial r^{2}+\partial \varepsilon / \partial r / r\right) / 2 k_{0} n_{0}-i k^{\prime \prime}\left(\partial^{2} \varepsilon / \partial t^{2}\right) / 2 \\
& +i k_{0} n_{2}|\varepsilon|^{2} \varepsilon-i k_{0} \rho \varepsilon / 2 n_{0} \rho_{c}-\sigma \rho \varepsilon / 2-U_{i} W(I)\left(\rho_{n t}-\rho\right) \varepsilon / 2 I
\end{aligned}
$$

where the evolution of the electron density $\rho$ follows the equation,

$$
\partial \rho / \partial t=W(I)\left(\rho_{n t}-\rho\right)+\sigma \rho|\varepsilon|^{2} / U_{i}
$$

where the terms on the right-hand side of Eq. (2) describe transverse beam diffraction, GVD, nonlinear self-focusing, absorption and defocusing due to the electron density $\rho$, and absorption by photo-ionization; the terms on the right-hand side of Eq. (3) are the generation of the plasma by photon ionization (involving multiphoton ionization and tunnel ionization) and avalanche (multiplication of the electrons in the laser field). In Eqs. (2) and (3), $k_{0}$ is the wave number in vacuum, $k^{\prime \prime}$ the second order dispersive coefficient, $\rho_{\mathrm{c}}$ the critical plasma density above which the plasma becomes opaque, $\rho_{\mathrm{nt}}$ the neutral atom density, $\sigma$ the crosssection for inverse bremsstrahlung, $U_{\mathrm{i}}$ the ionization potential, $I=\varepsilon \varepsilon^{*}$ the laser intensity and $W(I)$ the photoionization rate. Equations (2) and (3) can be solved simultaneously by the split-step method (Agrawal, 2007). The photoionization rate from Keldysh theory (Keldysh, 1965) can be calculated by the PPT (Perelemov-Popov-Terent'ev) model (Perelemov et al., 1966) which reduced to tunnelling (Ammosov et al., 1966) or multiphoton ionization models under certain conditions. An initial collimated Gaussian beam following a lens read as:

$$
\varepsilon(r, t, 0)=\varepsilon_{g} \exp \left(-r^{2} / w_{0}^{2}-t^{2} / t_{0}^{2}-i k r^{2} / 2 f\right)
$$

where $\varepsilon_{\mathrm{g}}$ is the peak amplitude of the incident electric field, $k=n_{0} k_{0}$ the wave number in the medium, $f$ the focal length of the lens, $t_{0}$ and $w_{0}$ are the half temporal width and the spatial radius of the $1 / e^{2}$ intensity (for), respectively. 


\subsection{Description of ionization}

Plasma generation and plasma defocusing are important dynamics in the filamentation formation. It is very important to construct the ionization model to describe the propagation of the intense pulse in filamentation. That is, how to calculate the parameter $W(I)$ in Eq. (3). Optical-field-ionization (OFI) covers both the multiphoton and tunnel regimes which we usually use the adiabaticity parameter $\gamma$ to distinguish with. For an atom with ionization potential $U_{\mathrm{i}}$ in a linearly polarized laser field with frequency $\omega_{0}$ and peak electric field $E, \gamma$ is defined as:

$$
\gamma=\omega_{0} \sqrt{2 m U_{i}} / e E
$$

where $e$ is the electron charge and $m$ the electron mass. OFI occurs in the tunnel regime when $\gamma \ll 1$ and in the multiphoton regime when $\gamma \gg 1$.

Keldysh's theory (Keldysh, 1965) and its subsequent developments (PPT model) (Perelemov et al., 1966) led to a general formula for OFI rates valid for any atom or ion with the orbital quantum number $l$ and its projection $m$ onto the quantization axis and charge state $Z$ :

$$
\begin{aligned}
& W\left(\omega_{0}, E\right)=\omega_{\text {a.u. }} \sqrt{6 / \pi}\left|C_{n^{*}, l^{*}}\right|^{2} f(l, m)\left(U_{i} / 2 U_{H}\right) \\
& \times A_{m}\left(\omega_{0}, \gamma\right)\left(2 E_{0} / E \sqrt{1+\gamma^{2}}\right)^{2 n^{*}-|m|-3 / 2} \exp \left(-2 E_{0} g(\gamma) / 3 E\right)
\end{aligned}
$$

where $U_{\mathrm{H}}$ is the ionization potential of hydrogen, $\omega_{\text {a.u. }}$ is defined by $\gamma=1$ from Eq. (5) for hydrogen or by $\hbar \omega_{\text {a.u. }}=2 U_{\mathrm{H}}$ :

$$
\begin{gathered}
\omega_{\text {a.u. }}=e E_{H} / \sqrt{2 m U_{H}} \approx 4.1 \times 10^{16} \mathrm{~s}^{-1} \\
E_{H}=e^{5} m^{2} / \hbar^{4}\left(4 \pi \varepsilon_{0}\right)^{3} \approx 5.14 \times 10^{11} \mathrm{~V} / \mathrm{m} \\
E_{0}=E_{H} \times\left(U_{i} / U_{H}\right)^{3 / 2}
\end{gathered}
$$

For a given atom, the dimensionless constant $C_{n^{*}, l^{*}}$

$$
\left|C_{n^{*} l^{*}}\right|^{2}=2^{2 n^{*}} / n^{*} \Gamma\left(n^{*}+l^{*}+1\right) \Gamma\left(n^{*}-l^{*}\right)
$$

is adapted from the formula well known for the hydrogen atom (Perelemov et al., 1966) by replacing the principal $n$ and orbital $l$ quantum numbers by their effective counterparts

$$
\begin{gathered}
n=Z\left(U_{i} / U_{H}\right)^{-1 / 2} \\
n^{*}=n-\delta l \\
l^{*} \approx n^{*}-1
\end{gathered}
$$

respectively, and

$$
\delta l=n-\left(U_{i} / U_{H}\right)^{-1 / 2}
$$

is the quantum defect. Here $\Gamma$ in Eq. (10) is the gamma function (Abramowitz \& Stegun, 1972). 
Other functions in Eq. (6) read as:

$$
\begin{gathered}
f(l, m)=(2 l+1) !(l+|m|) ! / 2^{|m|}(|m|) !(l-|m|) ! \\
f(0,0)=1 \\
A_{m}\left(\omega_{0}, \gamma\right)=4 \gamma^{2} / \sqrt{3 \pi}|m| !\left(1+\gamma^{2}\right) \\
\times \exp [-\alpha(k-v)] \Phi_{m}(\sqrt{\beta(k-v)}) \\
\Phi_{m}(x)=x^{2|m|+1} / 2 \times \int_{0}^{1} e^{-x^{2} t} t^{|m|} / \sqrt{1-t} d t \\
\beta(\gamma)=2 \gamma / \sqrt{1+\gamma^{2}} \\
\alpha(\gamma)=2\left[\sinh ^{-1} \gamma-\gamma / \sqrt{1+\gamma^{2}}\right]=\left\{\begin{array}{l}
2 \gamma^{3} / 3 \quad \text { when } \gamma \ll 1 \\
2 \log (2 \gamma-1) \text { when } \gamma \gg 1
\end{array}\right. \\
=\left\{\begin{array}{c}
1-\gamma^{2} / 10+9 \gamma^{4} / 280 \text { when } \gamma \ll 1 \\
3 / 2 \gamma \times(\log 2 \gamma-1 / 2) \text { when } \gamma \gg 1 \\
v=U_{i}\left(1+1 / 2 \gamma^{2}\right) / \hbar \omega_{0} \\
v_{0}=U_{i} / \hbar \omega_{0}
\end{array}\right.
\end{gathered}
$$

Single ionization of atoms in the fundamental state with $l=m=0, \mathrm{n}^{*}=\left(U_{\mathrm{i}} / \mathrm{U}_{\mathrm{H}}\right)^{-1 / 2}$ and $\left|C_{n^{*}, l^{*}}\right|^{2}=22^{2} / n^{*} \Gamma\left(2 \mathrm{n}^{*}\right)$ has explicit description for tunnel (ADK model) (Ammosov et al., 1986) and multiphoton limits.

When $\gamma \ll 1$, it is the tunnel ionization case where $A_{m}\left(\omega_{0}, \gamma\right) \rightarrow 1$, and we have

$$
W(E)=\omega_{\text {a.u. }} \sqrt{6 / \pi}\left|C_{n^{*}, l^{*}}\right|^{2}\left(U_{i} / 2 U_{H}\right)\left(2 E_{0} / E\right)^{2 n^{*}-3 / 2} \exp \left(-2 E_{0} / 3 E\right)
$$

We can see from Eq. (22) that the probability of tunnel ionization is independent of the frequency of the wave.

When $\gamma \gg 1$, it is the multiphoton case, by taking into account only the term $\kappa=K \equiv \bmod \left(v_{0}+1\right) \quad$ in the series for $A_{m}\left(\omega_{0}, \gamma\right)$, and by using the relation $v_{0}=U_{i} / \hbar \omega_{0}=E_{0} / 2 E \gamma$, we have

$$
\begin{aligned}
& W\left(\omega_{0}, E\right)=\omega_{a . u .}\left(4^{2 n^{*}} / \pi \sqrt{2}\right)\left|C_{n^{*}, l^{*}}\right|^{2}\left(U_{i} / 2 U_{H}\right) \\
& \times v_{0}^{2 n^{*}+2 K-3 / 2} e^{2 K-v_{0}} \Phi_{0}\left(\sqrt{2\left(K-v_{0}\right)}\right)\left(E / E_{0}\right)^{2 K}
\end{aligned}
$$

We can rewrite Eq. (23) as

$$
W\left(\omega_{0}, I\right)=\sigma_{K} I^{K}
$$


where

$$
\begin{gathered}
W\left(\omega_{0}, E\right)=\omega_{\text {a.u. }}\left(4^{2 n^{*}} / \pi \sqrt{2}\right)\left|C_{n^{*}, l^{*}}\right|^{2}\left(U_{i} / 2 U_{H}\right) \\
\times v_{0}^{2 n^{*}+2 K-3 / 2} e^{2 K-v_{0}} \Phi_{0}\left(\sqrt{2\left(K-v_{0}\right)}\right) / I_{0}^{K} \\
I_{0}=\varepsilon_{0} n_{0} c E_{0}^{2} / 2
\end{gathered}
$$

where $\varepsilon_{0}$ is the electric permittivity of free space, $c$ is the speed of light in vacuum.

\section{Simulation on gradient temperature (Song et al., 2008a; Song et al., 2008b)}

\subsection{Model of simulation}

In our simulation model, to simplify the calculation and hold the essential physical dynamic characteristics, we just only consider the fundamental mode (the spatial profile of which is not changing along propagation) of the coupled leaky modes propagating in the hollow fiber. We also neglect the interaction and energy transfer between the fundamental and high-order modes because the attenuation length of high-order modes is much smaller than that of the fundamental.

We use the standard nonlinear $(1+1)$ dimension Schrödinger equation to simulate and analyze the evolution dynamic of the pulse propagation both in temporal and spectra domain. The nonlinear Schrödinger equation for the electric field envelope $u(z, t)$ in a reference frame moving at the group velocity $v_{\mathrm{g}}$ takes the following form (assuming propagation along the $z$ axis) (Agrawal, 2007):

$$
\frac{\partial u}{\partial z}=-\frac{\alpha}{2} u+\frac{i \beta_{2}}{2} \frac{\partial^{2} u}{\partial T^{2}}+i \gamma\left[|u|^{2} u+\frac{i}{\omega_{0}} \frac{\partial}{\partial T}\left(|u|^{2} u\right)-T_{R} u \frac{\partial|u|^{2}}{\partial T}\right]
$$

The terms on the right hand side of the equation are the loss, second order dispersion, selfphase modulation, self-steepening and Raman scattering, respectively. Here $c$ is the speed of light in vacuum, $\omega_{0}$ the central angle frequency, $a$ the loss, $\beta_{2}$ the GVD (group velocity dispersion) and $T_{R}$ is related to the slope of the Raman gain spectrum. The nonlinear coefficient $\gamma=n_{2} \omega_{0} / c A_{\text {eff }}$ where $n_{2}$ is the nonlinear refractive index and $A_{\text {eff }}$ the effective cross section area of the hollow fiber. Equation (27) and the parameters in the equation characterize propagation of the fundamental mode.

The initial envelop of the pulse is in the following form (Tempea \& Brabec, 1998; Courtois et al., 2001), which is a simplification expression of Eq. (4):

$$
u(0, t)=\sqrt{\frac{2 P_{i n}}{\pi w_{0}^{2}}} \exp \left(-\frac{t^{2}}{t_{0}^{2}}\right)
$$

here $P_{\text {in }}$ is the peak power of the incident pulse, $w_{0}$ the spot size (for $1 / e^{2}$ intensity) of the fundamental beam (we assume that the beam focused on the entrance section of the hollow fiber matches the radius of the fundamental mode in our calculation mode), $t_{0}$ the half temporal width at the $1 / e^{2}$ points of the pulse intensity distribution.

Equations (27) and (28) can be solved by the split-step Fourier method (Agrawal, 2007) in which the propagation is broken into consecutive steps of linear and nonlinear parts. The 
linear part including loss and dispersion can be calculated in the spectrum domain by Fourier transform, while the nonlinear part which includes other terms on the right hand of Eq. (27) was solved in the time domain by Runge-Kutta method. The convergence of the solution can be easily checked by halving the step size to see if the calculation results are nearly unchanged.

Although the studies of filamentation in many gases have been focused by scientists and technologists (Akturk et al., 2007; Fuji et al., 2007; Dreiskemper \& Botticher, 1995), argon (Ar) is the most frequency used gas for generation of ultrashort intense femtosecond pulses. In simulation in this chapter, we employ Ar as the medium to reveal the essence of gradient temperature technology.

The loss and waveguide dispersion relations of the hollow fiber can be expressed as (Marcatili \& Schmeltzer, 1964):

$$
\begin{gathered}
\frac{\alpha}{2}=\left(\frac{2.405}{2 \pi}\right)^{2} \frac{\lambda^{2}}{2 a^{3}} \frac{v^{2}+1}{\left(v^{2}-1\right)^{1 / 2}} \\
\beta_{\text {waveguide }}=\frac{2 \pi}{\lambda}\left[1-\frac{1}{2}\left(\frac{2.405 \lambda}{2 \pi a}\right)^{2}\right]
\end{gathered}
$$

where $v$ is the refractive index ratio between the material of the hollow fiber (glass in our case) and the inner gas (argon in our case), $\lambda=\lambda_{0} / n$, the wavelength in the medium $\left(\lambda_{0}\right.$ the wavelength in vacuum), $a$ the bore radius of the hollow fiber. The propagation constant $\beta$ icluding contributions from both waveguide part (Eq. (30)) and material part:

$$
\beta_{\text {material }}=\frac{\omega}{c} n
$$

The relation between propagation constant $\beta$ and $\mathrm{m}$ order dispersion coefficient $\beta_{\mathrm{m}}$ is:

$$
\beta_{m}=\left(\frac{d^{m} \beta}{d \omega_{m}}\right)_{\omega=\omega_{0}}
$$

For gases, the refractive index is a function of both temperature and pressure (Lehmeier, 1985):

$$
n=\left(2 \frac{n_{0}^{2}-1}{n_{0}^{2}+2} \frac{p T_{0}}{p_{0} T}+1\right)^{1 / 2}\left(1-\frac{n_{0}^{2}-1}{n_{0}^{2}+2} \frac{p T_{0}}{p_{0} T}\right)^{-1 / 2}
$$

For argon, at the standard condition $(\mathrm{T}=273.15 \mathrm{~K}, \mathrm{p}=1 \mathrm{~atm})$, we have (Dalgarno \& Kingston, 1960):

$$
n_{0}^{2}-1=5.547 \times 10^{-4}\left(1+\frac{5.15 \times 10^{5}}{\lambda_{0}^{2}}+\frac{4.19 \times 10^{11}}{\lambda_{0}^{4}}+\frac{4.09 \times 10^{17}}{\lambda_{0}^{6}}+\frac{4.32 \times 10^{23}}{\lambda_{0}^{8}}\right)
$$

In the above equations, $p$ is the pressure, $p_{0}$ the pressure at normal conditions $(1 \mathrm{~atm}), T$ the temperature, $T_{0}$ the temperature at normal conditions $(273.15 \mathrm{~K}), n$ the refractive index of 
the medium, and $n_{0}$ the refractive index of the medium at normal conditions ( $\mathrm{T}=273.15 \mathrm{~K}$, $\mathrm{p}=1 \mathrm{~atm})$. In Eq. (34), the unit of $\lambda_{0}$ is $\AA\left(10^{-10} \mathrm{~m}\right)$.

Before we do the simulations of the evolution of the pulse under gradient temperature, we first check the effect of the temperature on the hollow fiber and the medium (Ar as in our case) qualities such as loss, refractive index, etc. Figures 2 and 3 show the loss and refractive index as a function of the temperature. They all keep nearly constant during the interval from $300 \mathrm{~K}$ to $600 \mathrm{~K}$. We can conclude that compared with room temperature, higher temperature does not introduce extra attenuation during the pulse propagation.

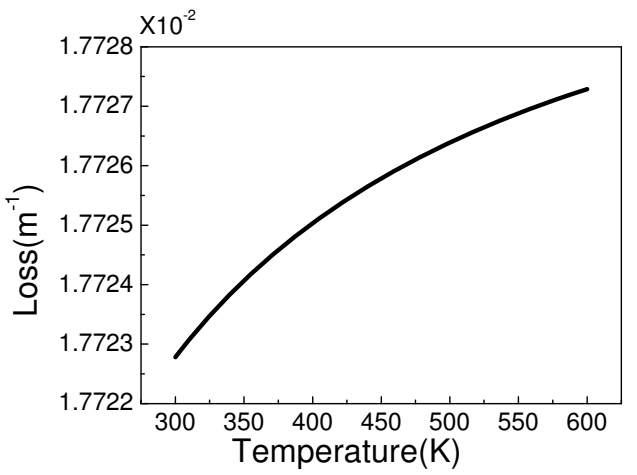

Fig. 2. Loss as a function of temperature for Ar in hollow fiber (bore diameter $500 \mu \mathrm{m}$, pressure1 atm).

To simplify and catch the essence physics process, we define a factor TF which represents the gas gradient temperature factor through the ideal gas equation: $T F=p T_{0} / p_{0} T$. It is obvious to see that the factor TF is proportional to the gas density (proportional to the gas pressure while inversely proportional to the gas temperature). When the gas pressure is 1 atm, the gradient factor TF is 1 for $300 \mathrm{~K}$, and 0.5 for $600 \mathrm{~K}$.

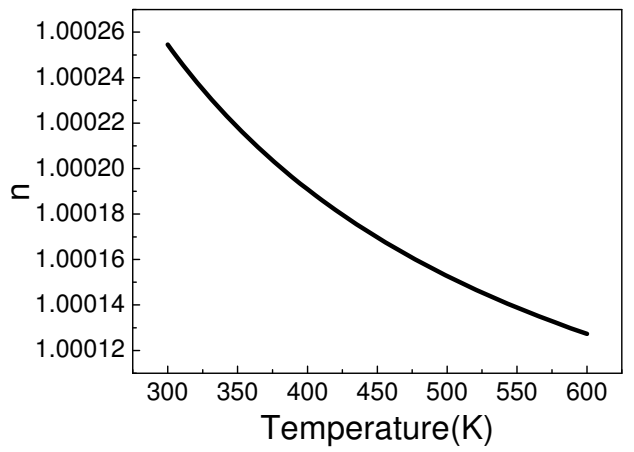

Fig. 3. Refractive index as a function of temperature for Ar at $1 \mathrm{~atm}$.

The nonlinear refractive index and GVD are both proportional to the factor TF (Mlejnek et al., 1998): 


$$
\begin{gathered}
n_{2}=4.9 \times 10^{-23} \times \mathrm{TF}\left(\mathrm{m}^{2} / \mathrm{W}\right) \\
\beta_{2}=2.6 \times 10^{-29} \times \mathrm{TF}\left(\mathrm{s}^{2} / \mathrm{m}\right)
\end{gathered}
$$

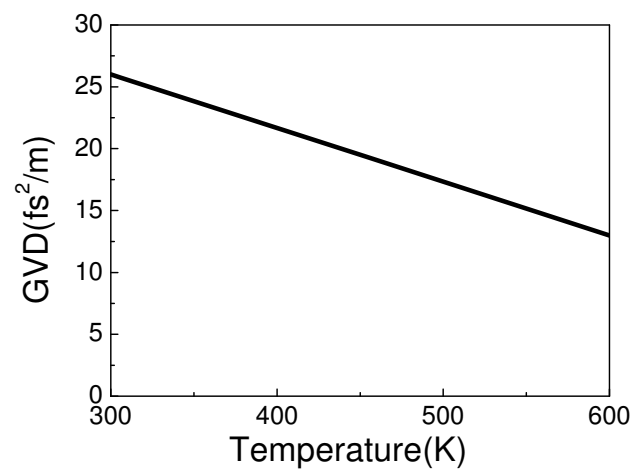

Fig. 4. GVD as a function of temperature (bore diameter $500 \mu \mathrm{m}$, pressure1 atm).

Now we can calculate the GVD and nonlinear refractive index by Eqs. (30)-(36). The results are shown in Figs. 4 and 5. As we can see from these figures, a higher temperature at $600 \mathrm{~K}$ decreases both the GVD and nonlinear refractive index $n_{2}$ by a factor of 2 for the room temperature $300 \mathrm{~K}$. The decreasing GVD gives the pulse a chance to slow down the pulse broadening in time domain; while the decreasing nonlinear refractive index increases the critical power for self-focusing $P_{c}$ (see Eq. (1)). Therefore, at a higher temperature, the pulse broadening in time domain slows down and $P_{\mathrm{c}}$ is higher. If the tube is sealed and is locally heated at the entrance, and cooled at the exit end, the gas temperature gradient will be formed along the tube and so will the nonlinear refractive index. $P_{\mathrm{c}}$ at the hot side of the tube (entrance) will be higher than the cold end (exit end), like in the case of gradient pressure (see Fig. 1).

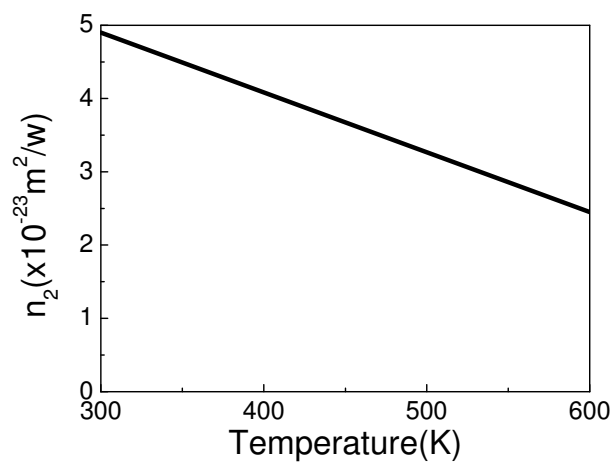

Fig. 5. Nonlinear refractive index as a function of temperature (pressure $1 \mathrm{~atm}$ ). 


\subsection{Spectrum broadening}

As the incident pulse propagating along the hollow fiber filled with argon, the peak power of the pulse is continuously decreasing due to the dispersion and loss. However, the decreasing temperature along the fiber provides a gradually increasing nonlinear coefficient which partly compensates the decreasing peak power, the spectrum broadening can go on till the end of the tube. For the argon gas at atmospheric pressure and temperature of $600 \mathrm{~K}$, $P_{\mathrm{c}}$ is $4.2 \mathrm{GW}$; while for the room temperature, $300 \mathrm{~K}$, it is $2.1 \mathrm{GW}$, i.e. the critical power for $600 \mathrm{~K}$ is twice of that for $300 \mathrm{~K}$. This means that the energy of the incident pulse will be allowed twice higher as that of the pulse under room temperature for the same pulse width. We did the simulation on the spectrum broadening for the uniform and gradient temperature cases in the hollow fiber. The bore diameter of the hollow fiber was $500 \mu \mathrm{m}$ and the length of the fiber was $60 \mathrm{~cm}$. The temperature conditions are: condition 1: uniform room temperature $(\mathrm{T}=300 \mathrm{~K})$; condition 2 : temperature linearly decreasing from $600 \mathrm{~K}$ to $300 \mathrm{~K}$ along the hollow fiber; condition 3: temperature linearly decreases from $600 \mathrm{~K}$ to 300 $\mathrm{K}$ in the first half and increases from $300 \mathrm{~K}$ to $600 \mathrm{~K}$ in the second half of the fiber, i.e., the triangle temperature. The incident peak power of the pulses was set to be $2 P_{\mathrm{c}}$ and the pressure was $0.2 \mathrm{~atm}$, thus, for a $30 \mathrm{fs}$ pulse, the incident pulse energy should be $0.6 \mathrm{~mJ}$ and $1.2 \mathrm{~mJ}$, for room temperature $300 \mathrm{~K}$ (uniform case) and $600 \mathrm{~K}$ (gradient temperature case), respectively.

By solving Eq. (27) coupled with the initial condition in Eq. (28), we obtained the spectra and phases of the output pulses under the above three conditions, which are shown in Figs. 6 and 7. It is obvious that the output spectrum bandwidth of the pulse increases from 250 $\mathrm{nm}$ (about $675 \mathrm{~nm}$ to $925 \mathrm{~nm}$, uniform temperature case) to $350 \mathrm{~nm}$ (about $625 \mathrm{~nm}$ to $975 \mathrm{~nm}$, linear and triangle gradient temperature case). However, the triangle shaped gradient temperature does not seem to make visible difference from the linear gradient temperature case. We also plot the spectrum evolution of the triangle shaped temperature in Fig. 7(b). We can see that the spectrum starts to expand at about $20 \mathrm{~cm}$ and the profile collapses along the fiber. We will discuss on the spectrum broadening quantatively in the following subsection.
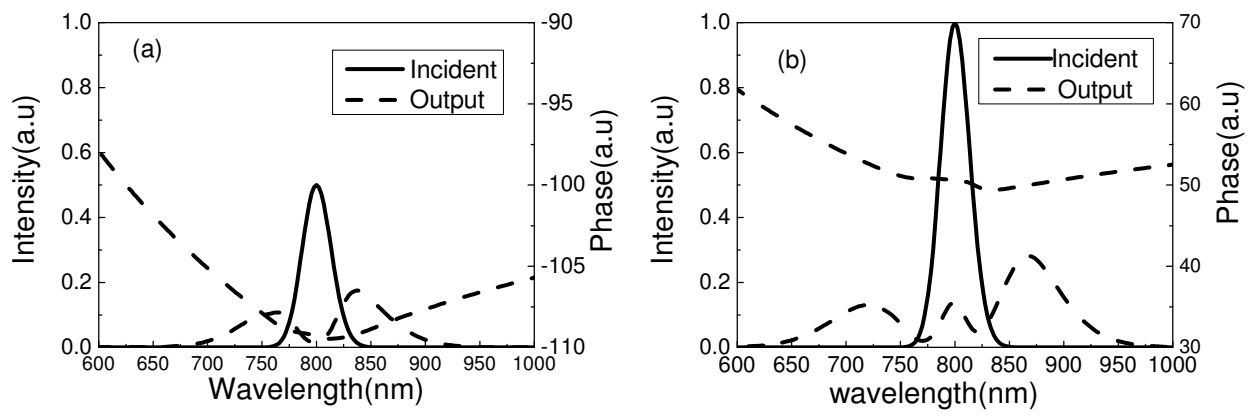

Fig. 6. Spectrum \& phase for (a) uniform temperature (300 K), (b) linear gradient temperature ( $600 \mathrm{~K}$ to $300 \mathrm{~K}$ ) and. Other conditions: bore diameter of the hollow fiber: 500 $\mu \mathrm{m}$, fiber length: $60 \mathrm{~cm}$, filled argon gas pressure: $0.2 \mathrm{~atm}$, incident pulse width: $30 \mathrm{fs}$, pulse energy: $0.63 \mathrm{~mJ}$ for the uniform case and $1.26 \mathrm{~mJ}$ for the gradient case. 


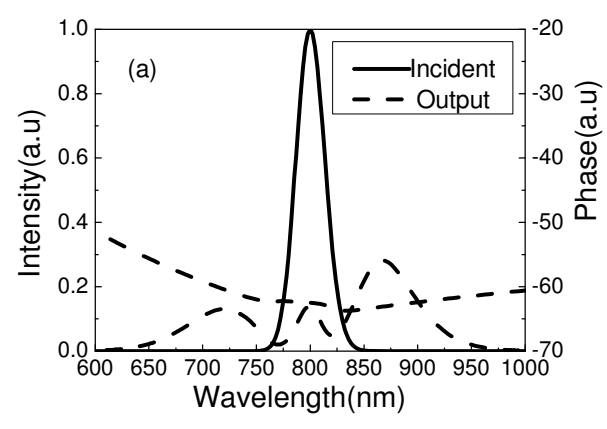

(b)

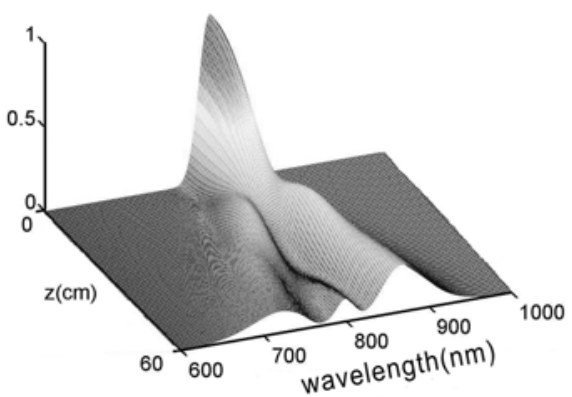

Fig. 7. (a) Spectrum \& phase (b) Spectra evolution for triangle gradient temperature $(600 \mathrm{~K}$ to $300 \mathrm{~K}$ to $600 \mathrm{~K})$. Other conditions: bore diameter of the hollow fiber: $500 \mu \mathrm{m}$, fiber length: $60 \mathrm{~cm}$, argon gas pressure: $0.2 \mathrm{~atm}$, incident pulse width: $30 \mathrm{fs}$, pulse energy: $1.26 \mathrm{~mJ}$.
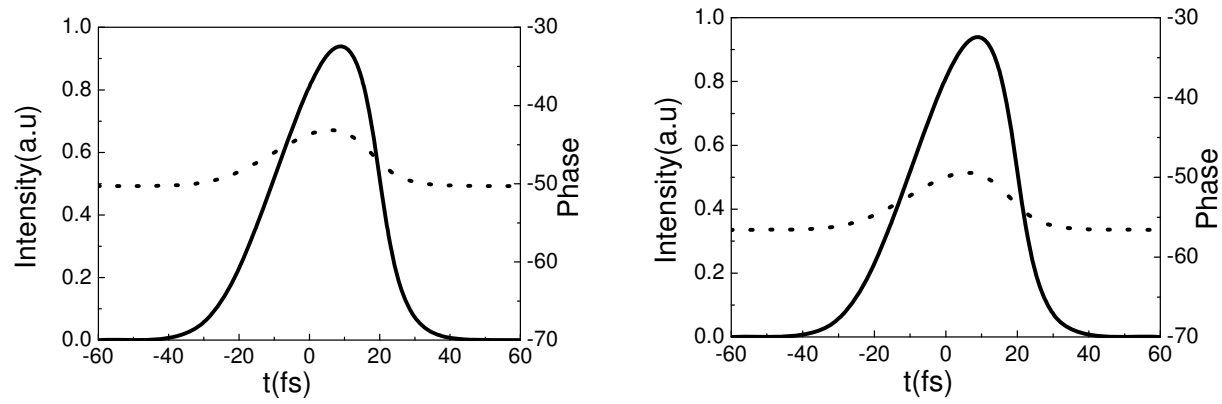

Fig. 8. Pulse profiles \& phases for the (a) linear gradient and (b) triangle gradient temperature in Fig. 6 (b) and 7 (a).

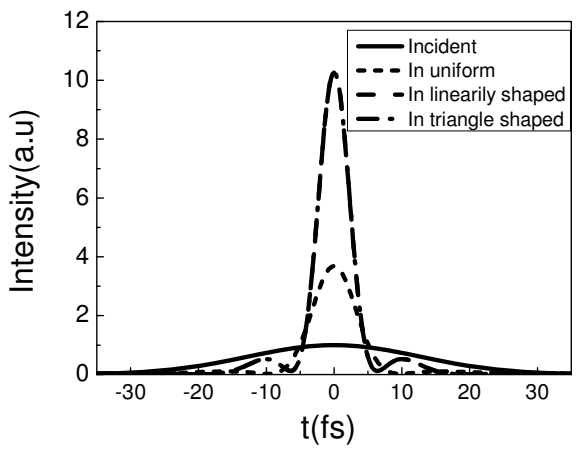

Fig. 9. Pulses profiles after ideal compression (spectra are shown in Figs. 6 and 7(a) respectively). 
The output pulse profiles and phases of the linear and triangle gradient temperature are shown in Figs. 8(a) and 8(b) respectively. Still, we cannot see much difference between the linear and triangle cases for the output pulse. The transform limited pulse after ideal compression for the three conditions are shown in Fig. 9. The pulse width after ideal compression is $5 \mathrm{fs}$ in the gradient temperature case (both linear and triangle gradient cases), which is $2 / 3$ of pulse width in the uniform temperature case (7.5 fs). In addition, the pulse energy we can obtain in the gradient temperature scheme is twice higher as that in the uniform temperature scheme.

\subsection{Discussions on spectrum broadening}

When a pulse propagates through a Kerr medium whose length is $L$, the spectrum broadening $S_{p}$ of the pulse is approximately determined by the integral below (Agrawal, 2007):

$$
S_{p}=\int_{0}^{L} n_{2}(z) P(z) d z
$$

where $n_{2}(z)$ is the nonlinear refractive index at position $z, P(z)$ the peak power of the pulse at position z. We use Eq. (37) to discuss the spectrum broadening comparing with the simulation we did in the above subsection.

First, this integral can approximately determinate the spectrum broadening quantatively. If we take $n_{2}(z) P(z)$ as a variable and set it equal everywhere along the medium, the nonlinear Schrödinger equation (Eq. (27)) is actually the same in every $z$ of the medium. The result is that the final pulse temporal and spectral profiles (normalized with themselves) are the same, which means that they are only different with intensity.

Second, from the integral we can see that the spectrum broadening will not be much broader in the gradient temperature case than that in the uniform case. But from the energy point, we can see that the incident energy will be allowed twice higher than uniform temperature. This is a big priority of gradient temperature. Our intention is to achieve not only ultrashort but also intense pulses. The energy is also a main final object which we focus on.

Third, from the integral in Eq. (32) we can deduce that the spectrum broadening in triangle gradient will be almost the same as that in the linear gradient case. This is true and can be verified by our simulation results (see Figs. 6 (b) and 7 (a)). In fact, the difference of linear and triangle gradient scheme excluding real experimental conditions in simulation is small. Their different effects can be seen from experiments more obviously. Triangle gradient scheme's priority is that this design gives even better pulse compression, avoids cyclic compression stages, and therefore limits the energy loss as shown in Ref (Couairon et al., 2005). From ideal theoretical point, these two schemes have almost the same ability of spectrum broadening. From the experimental point, triangle scheme has priority to linear project and it is a little more complex. Although this experimental conclusion is obtained from gradient pressure scheme, we can expect the same results in gradient temperature case.

\subsection{Ideal gradient line shape}

In the above simulation, we set the input pulse peak power related to the critical selffocusing power $P_{\mathrm{c}}$. Inversely, we can derive an ideal gradient shape for a giving pulse, which means that at every step of evolution, we change the temperature so as to make the 
pulse's peak power equals to the critical power of self-focusing. Figure 10 shows the ideal gradient shape for a $30 \mathrm{fs}, 0.1 \mathrm{~mJ}$ incident pulse. The TF differential increases along the fiber, which implies that the peak power of the pulse along the tube drops faster and faster during the evolution. If we can realize such a gradient temperature, we can avoid multi-filament formation everywhere along the tube. In fact, in the linear gradient shape case, a moderately increasing the length of the tube (corresponding to decreasing the slope of the linear line) or decreasing the peak power of the input pulse will avoid the self-focusing or filament formation everywhere along the tube and the result of the spectrum broadening is still much broader than the uniform temperature case.

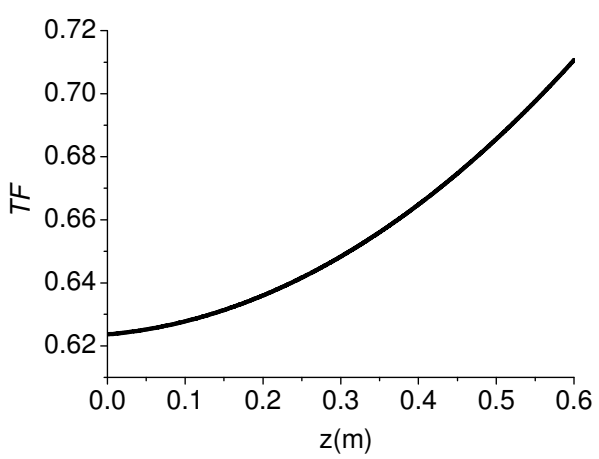

Fig. 10. Ideal gradient shape for $0.1 \mathrm{~mJ}$.

\section{Experimental results (Cao et al., 2009)}

As we mentioned in the introduction, spectrum broadening through filamentation was one of the most extremely simple and robust techniques to generate intense few to monocycle pulses with less sensitivity to the experiment conditions. In real experiments, an aperture (Cook et al., 2005), rotating lens, anamorphic prisms, circular spatial phase mask (Pfeifer et al., 2006), periodic amplitude modulation of the transverse beam profile (Kandidov et al., 2005), introducing beam astigmatism (Fibich et al., 2004) or incident beam ellipticity (Dubietis et al., 2004) in the laser beam prior to focusing have been used to stabilize the pointing fluctuations of a single filament. In the previous section, we show the priority of the gradient temperature scheme by theoretical simulation. In this section, we will verify the robustness of this scheme by showing the experimental results.

We show our experimental setup in Fig. 11. The laser pulse was produced from a set of conventional chirped pulse amplification (CPA) Ti: sapphire laser system. This laser system produced linearly polarized pulses of $37 \mathrm{fs}$ pulse at the central wavelength of $805 \mathrm{~nm}$. The energy of the pulses was $2 \mathrm{~mJ}$ and the repetition rate was $1 \mathrm{kHz}$. The beam diameter of the pulses was $10 \mathrm{~mm}$ (at $1 / e^{2}$ of the peak intensity). In this experiment, four silver mirrors were used to couple the amplified pulses into the sealed silica tube, where M1, M2 and M3 were the plane mirrors and FM1 was a concave mirror with a $1.7 \mathrm{~m}$ radius of curvature. A hard aperture $\mathrm{A} 1$ as an attenuator and a beam profile shaper was inserted in front of the concave mirror FM1. The output pulse was focused by a concave mirror, FM2, into a pulse compression system consisting of two negative dispersion mirrors, CM1 and CM2. The negative dispersion mirrors were rectangles of size $10 \times 30 \mathrm{~mm}^{2}$. Each reflection contributed 
a GDD of $50 \mathrm{fs}^{2}$ within wavelength area of $680 \sim 1100 \mathrm{~nm}$. The pulse after compression was reflected by plane mirrors, M4 and M5, then through a beam split mirror, BS1, into SPIDER. The Ar gas filled in the tube was controlled and monitored to be below the maximum pressure of $3 \mathrm{~atm}$, because a higher gas pressure may blow up the windows of the tube. The focal point in the tube was measured as $47 \mathrm{~cm}$ from the input window. The spot size of the focused pulse was $100 \mu \mathrm{m}$. To make a temperature gradient along the propagation of the pulse, a $20 \mathrm{~cm}$ heating length furnace was used to heat the tube. The $100 \mathrm{~cm}$ long hightemperature and high-gas-pressure resistance silica tube with the inner diameter of $25 \mathrm{~mm}$ was sealed off with two 1-mm thick fused silica Brewster windows. The tube was inserted into the transverse center of the furnace. Two ends of the tube were cooled by air. To avoid the expansion of the tube and make the furnace easy to move along the tube, between the external side of the tube and the internal side of the furnace, there was a $2 \mathrm{~mm}$ wide gap. The temperature of the furnace was controlled by a temperature controller between $25^{\circ} \mathrm{C}$ and $500{ }^{\circ} \mathrm{C}$ with a $\pm 5^{\circ} \mathrm{C}$ precision. It should be noted that the temperature we mention in the following text in this section is the temperature at the longitude center of the furnace. With this configuration, the temperature at the heating point could be increased from $25^{\circ} \mathrm{C}$ to $500^{\circ} \mathrm{C}$ within 35 minutes. The above experimental setup is the same as that was used in broadening the spectrum through filamentation, expecting for the additional furnace. Therefore, an additional furnace and temperature controller are sufficiently easy to modify the traditional filamentation setup to our experimental setup.

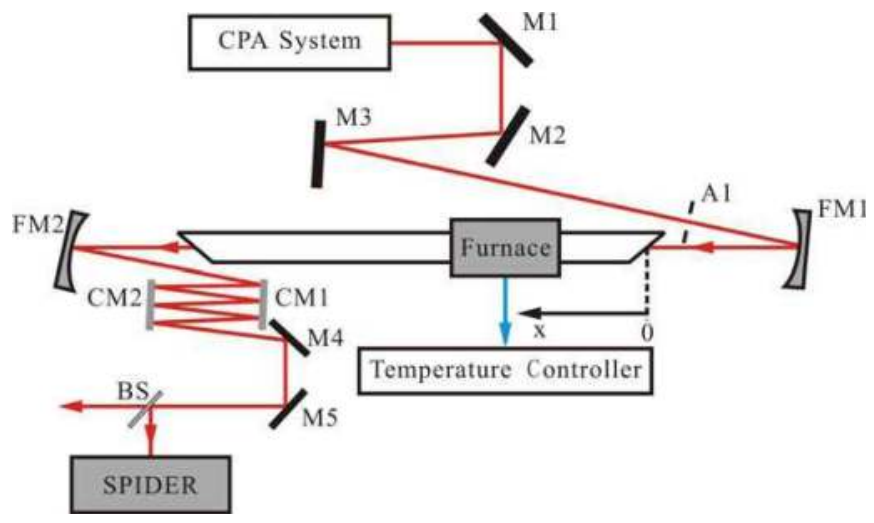

Fig. 11. The schematic of the experimental setup

To know the actual temperature distribution inside the tube, we inserted a thermistor and moved it along the tube to measure the temperature. The measured temperature distribution at a maximum central temperature of $500^{\circ} \mathrm{C}$ is shown in Fig. 12. The temperature rapidly drops down to the room temperature outside the furnace, so that the temperature distribution is of a triangular shape, with the temperature gradient of about $2403{ }^{\circ} \mathrm{C} / \mathrm{m}$. According to our simulation results and discussions in the former section, the priority of the triangle gradient is that it gives an even better pulse compression, avoids cyclic compression stages, and limits the energy loss. As the temperature is distributed along the tube, there should be a gas flowing from the hot to the cool position. However, in the experiment, the temperature variation was a very slow process. We did not observe the instability caused by the gas turbulence. In general, the radial thermal distribution could 
also cause thermal lensing effect. In our case, because the inner tube diameter was only 25 $\mathrm{mm}$, the radial temperature difference between the wall and the center was measured to be only $2-3^{\circ} \mathrm{C}$, so that the thermal lensing effect could be neglected.

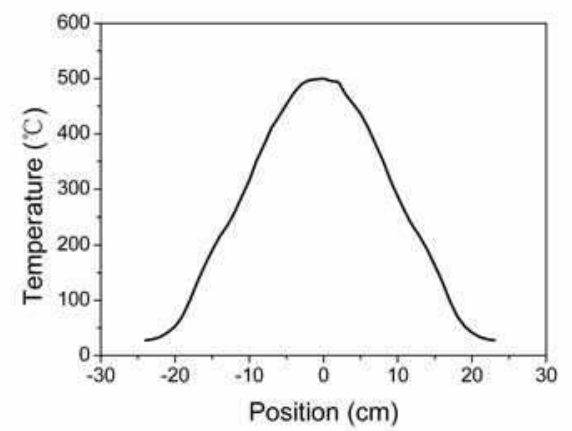

Fig. 12. Temperature distribution along the tube when the temperature at the furnace central (zero point at $x$-axis) is $500^{\circ} \mathrm{C}$.

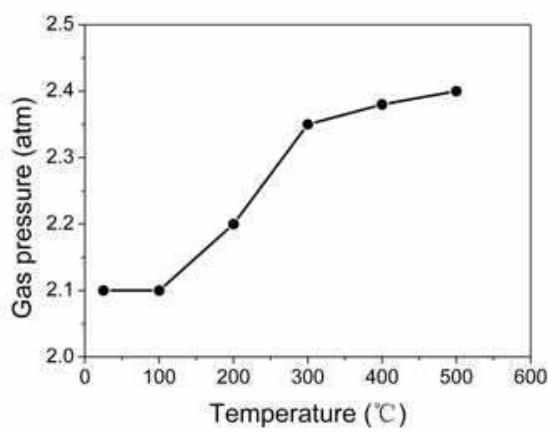

Fig. 13. Measured gas pressure as a function of the heated temperatures when the initial gas pressure is $2.1 \mathrm{~atm}$

As for the sealed tube, the gas pressure in total should be uniform and increase with the temperature. The measured gas pressure as a function of the heated temperatures when the initial gas pressure is $2.1 \mathrm{~atm}$ is shown in Fig. 13. Generally speaking, the influence of the pressure and temperature should be separately examined. However, since we just wanted to investigate the filamentation process in a sealed tube with the change of the temperature, we did not attempt to separate the temperature and pressure effects in our experiment. Moreover, the pressure change within $100{ }^{\circ} \mathrm{C}$ was only a few percent. This small change does not introduce noticeable difference in the material parameters for argon gas such as $\mathrm{GVD}, n_{2}$, and the average electron collision time.

\subsection{Filament controll and spectrum broadening by gradient temperature}

To check the influence of the temperature, we changed the local temperature in the tube and measured the beam pattern and the broadened spectrum. The beam pattern was taken by an 
ordinary digital camera looking at the surface of a white paper positioned at the plane orthogonal to the beam path and $3 \mathrm{~m}$ away from the exit window of the tube and the broadened spectrum was measured by a spectrometer (Ocean Optics, SD2000). When the furnace was turned off, the temperature was kept at room temperature $25{ }^{\circ} \mathrm{C}$ inside the tube, and the gas density was uniform along the tube. Pulses with energy of $1.2 \mathrm{~mJ}$ (32.4 GW peak power, about 6.5 times higher than the critical power at $2.1 \mathrm{~atm})$ after the aperture A1 were coupled into the tube, and output pulse energy of the pulses was $1.1 \mathrm{~mJ}$. A single filament began to appear at $3 \mathrm{~cm}$ before the focal point and the filament was about $40 \mathrm{~cm}$ long at 1.7 atm. By increasing the gas pressure to above $2.1 \mathrm{~atm}$, the single filament broke into multiple filaments, as is shown in Fig. 14(a). The inserted picture is the output beam profile of the multi-filament in the far field, where three filament spots can be identified. The interactions among multi-filament result in shot-to-shot fluctuations in the filamentation pattern. As the temperature was increased to $200^{\circ} \mathrm{C}$, the gas pressure in the tube was increased to $2.2 \mathrm{~atm}$, a little higher than that at $25^{\circ} \mathrm{C}$ (see Fig. 13). Although the heated gas will flow to the cool end and be kept at the same temperature in a long term, the gas temperature of the exit end of the tube was found still $25^{\circ} \mathrm{C}$. When the furnace was increased to $200{ }^{\circ} \mathrm{C}$, the mult-filament turned to become a single filament, as shown in Fig. 14(b). There was only one single filament that has a good beam profile. Further increasing the temperature to $300{ }^{\circ} \mathrm{C}$ or higher, the single filament collapsed and disappeared, as shown in Fig. 14(c). Although the gas pressure was also increased at the same time, the higher gas pressure was caused by the accelerated activity of the gas atoms, but not because of the increase of the number of the gas atoms. Gas atoms moved from the position of higher temperature to that of lower temperature, which resulted in that the gas density was lower at the entrance and higher at the end of the filament. Higher self-focusing critical power $P_{c}$ induced by the higher gas

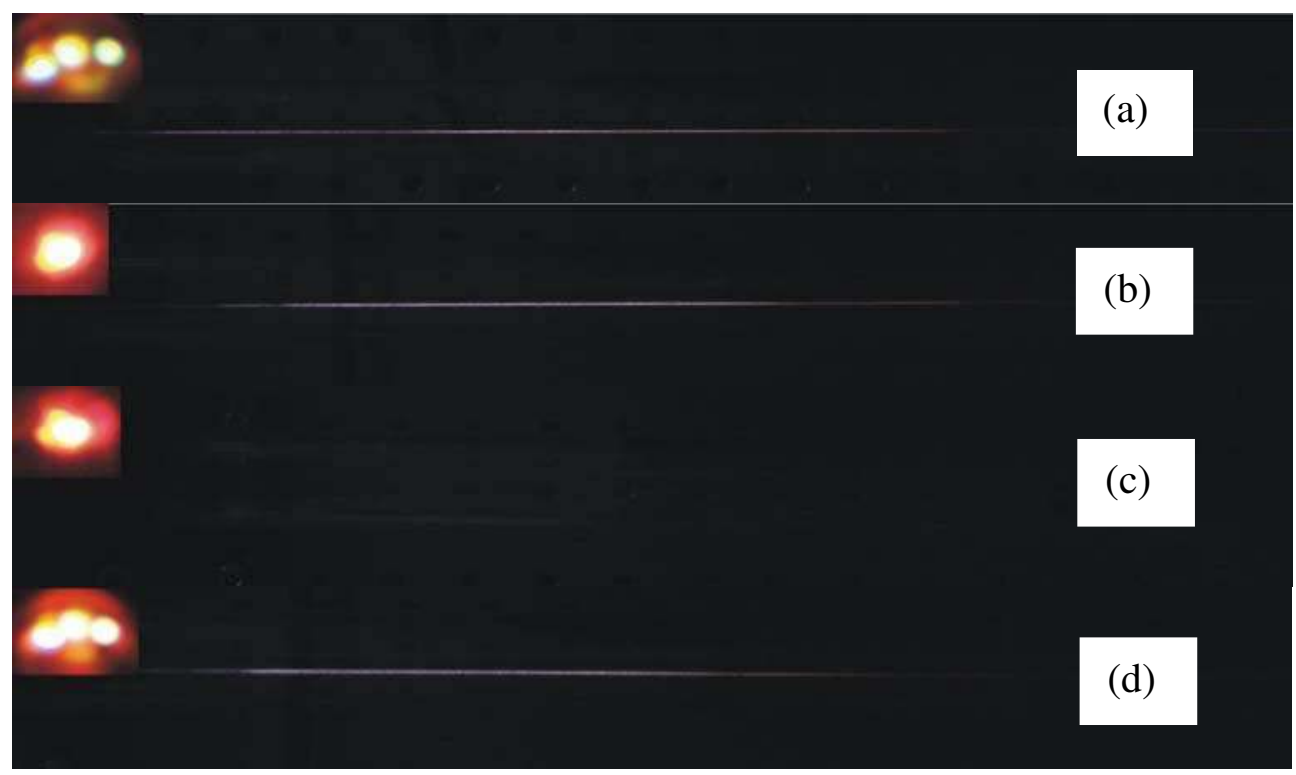

Fig. 14. Filament pattern at temperature of (a) $25^{\circ} \mathrm{C}$; (b) $200^{\circ} \mathrm{C}$; (c) $300^{\circ} \mathrm{C}$; (d) $25^{\circ} \mathrm{C}$. The inserted pattern in every picture is the output beam profile. 
density was effective to avoid the occurrence of the multi or even single filamentation. Inversely, by decreasing the temperature from $300{ }^{\circ} \mathrm{C}$ to the initial room temperature $25^{\circ} \mathrm{C}$, multi-filament appeared gradually, which is shown in Fig. 14(d). It was almost the same as in the initial state (Fig. 14(a)) of our experiment.

At the temperate of $25^{\circ} \mathrm{C}$ and input pulse energy of $1.2 \mathrm{~mJ}$, we measured the output spectra at different gas pressure. The results are shown in Fig. 15. The output spectra toward the short wavelength became wider with the increase of gas pressure, which resulted from the increase of the number of the filled gas atoms. At the gas pressure of $2.1 \mathrm{~atm}$, multi-filament was formed in the gas-filled tube.

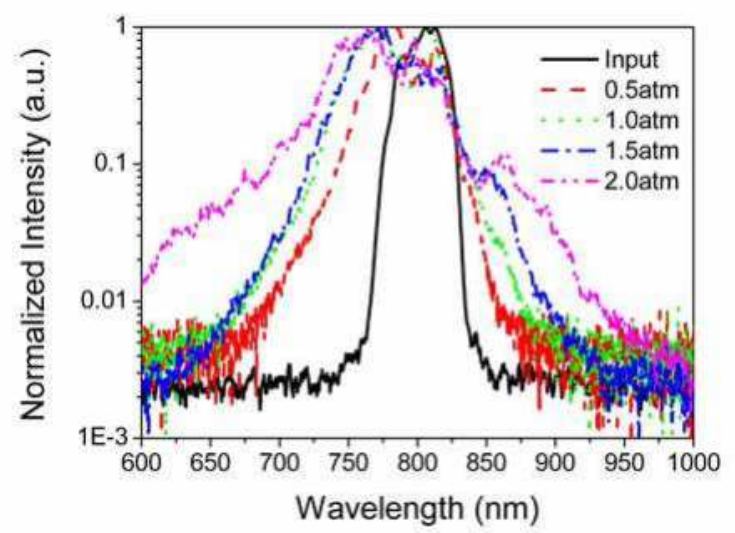

Fig. 15. Spectra at different gas pressures with the input pulse energy of $1.2 \mathrm{~mJ}$ and the heated temperature of $25^{\circ} \mathrm{C}$

Fig. 16 shows the evolution of output spectra at different temperatures of the entrance of the filament, when the gas pressure was $2.1 \mathrm{~atm}$ and incident pulse energy was $1.2 \mathrm{~mJ}$. It can be seen that the spectral width was broadened to about twice as that of the incident spectrum. For a single filament at $200{ }^{\circ} \mathrm{C}$, the spectrum broadening is due to an increasing phase contribution from ionization-induced spectrum broadening and interaction with the plasma. Whereas, in the case of non-filament at above $300^{\circ} \mathrm{C}$, the spectrum broadening is due to the dominant self-phase modulation (SPM) rooted from $n_{2}$, which becomes weak with the increase of the temperature. The further increasing of the temperature results only in a narrower broadened spectrum. When the filament disappears at high temperature, it means that the self-focusing critical power is high.

Therefore, we can increase the input pulse energy up to the new self-focusing critical power. The final results are shown in Fig. 17. At the temperature of $25^{\circ} \mathrm{C}$ and incident pulse energy of $1.2 \mathrm{~mJ}$, filament was formed at $2.1 \mathrm{~atm}$, shown as the point $\mathrm{A}$ in Fig. 17. Then, when the temperature at the entrance of the filament was increased to $300{ }^{\circ} \mathrm{C}$, the filament disappeared, shown as the point B in Fig. 17. After increasing the pulse energy from $1.2 \mathrm{~mJ}$ to $1.54 \mathrm{~mJ}$ at $300{ }^{\circ} \mathrm{C}$, the filament appeared again, shown as the point $\mathrm{C}$ in Fig. 17. After increasing the temperature from $300^{\circ} \mathrm{C}$ to $400{ }^{\circ} \mathrm{C}$ at $1.54 \mathrm{~mJ}$, the filament disappeared again, shown as the point D in Fig. 17. It indicates that the filament can appear or disappear by increasing the temperature and input pulse energy in turn. Meanwhile, if the temperature 


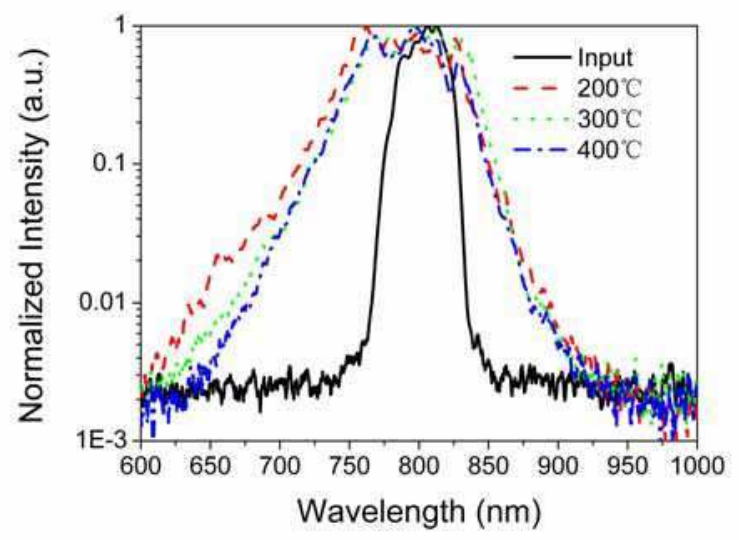

Fig. 16. Spectra at different temperatures with the input pulse energy of $1.2 \mathrm{~mJ}$ and the initial gas pressure of $2.1 \mathrm{~atm}$

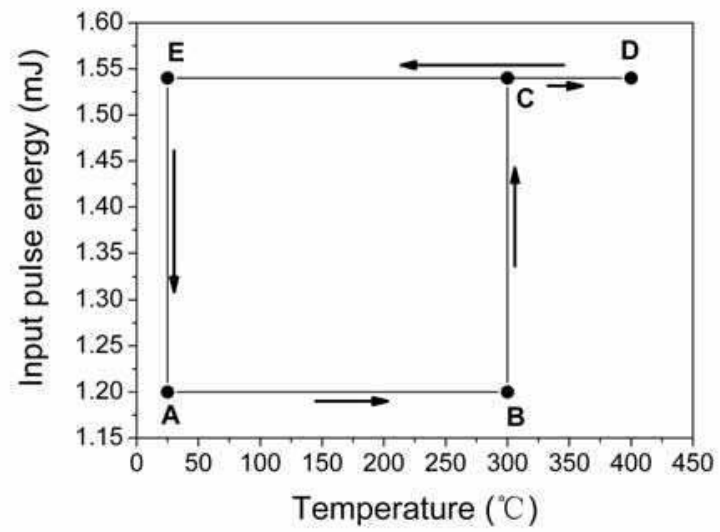

Fig. 17. The cycle between filament and no-filament by changing the temperature and input pulse energy in turn. Filament appears at points A, C, and E and disappears at points B and D. was decreased to $25{ }^{\circ} \mathrm{C}$ at the incident pulse energy of $1.54 \mathrm{~mJ}$, the filament also appeared, shown as the point E in Fig. 17, and further when the pulse energy was decreased to $1.20 \mathrm{~mJ}$, the filament was the same as in the initial state. The above experimental results indicate that the filament can be controlled by adjusting the local self-focusing critical power by the temperature, although the broadened spectrum narrows with the increase of the temperature. More incident pulse energy can be allowed in the tube at the higher local temperature. The presented method is simple and feasible to operate with only a heating furnace, without continuing consumption of expensive gases comparing with the gradient pressure scheme. 


\subsection{Self compression in temperature controlled filamentation}

The filamentation of intense femtosecond laser pulses will lead to a remarkable pulse selfcompression. The filamentation of ultrashort laser pulses is a banlance between the beam self-focusing by the optical Kerr effect, beam defocusing due to the plasma, pulse selfsteepening, and beam diffraction. The propagating pulse suffers significant reshaping in both time and space domain. This reshaping process will lead to the self compression of the intense femtosecond pulse. In this part, we investigate the self compression of the femtosecond pulse propagation in temperature controlled filamentation. We start fromed $0.7 \mathrm{~mJ}$ incident pulse with $25^{\circ} \mathrm{C}$ and $2 \mathrm{~atm}$. In this condition, it could only form a very short filament at the focal point. The output pulse reduced from $35 \mathrm{fs}$ to $23.5 \mathrm{fs}$ due to self compression, with a Fourier transform limit of 16 fs (see Fig. 18).

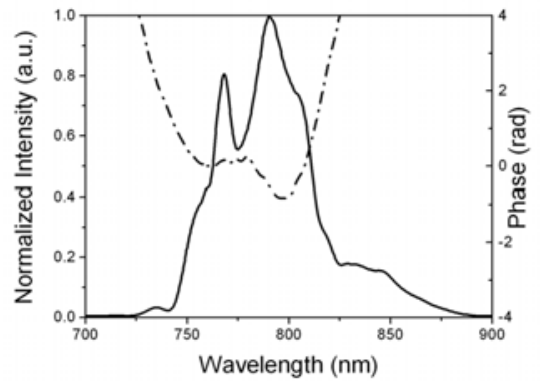

(a)

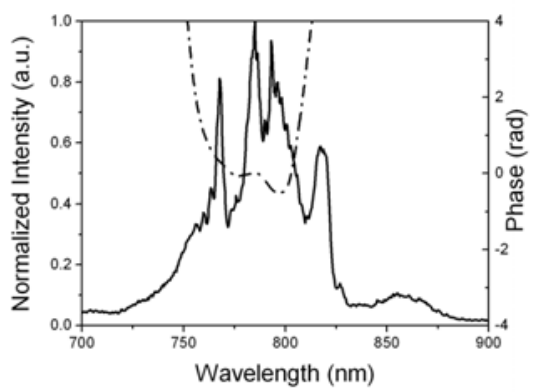

(c)

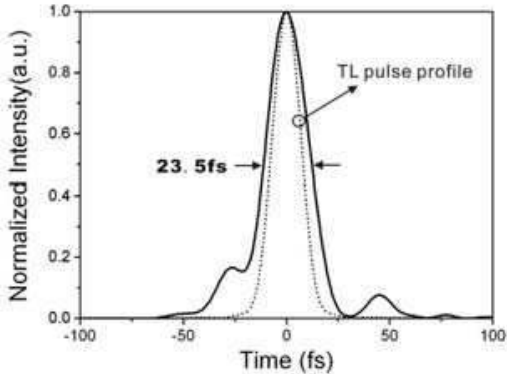

(b)

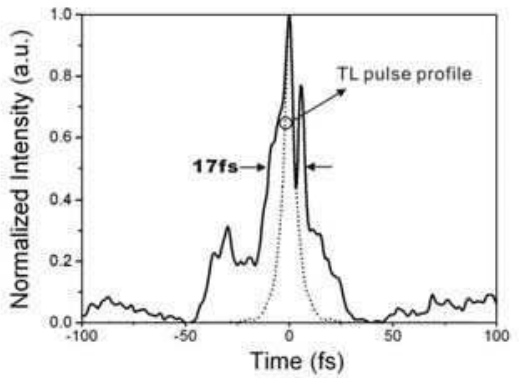

(d)

Fig. 18. The spectra and phases of (a) $0.7 \mathrm{~mJ}$, (c) $0.8 \mathrm{~mJ}$ incident pulse at $25^{\circ} \mathrm{C}$. Pulse profiles after self compression and Fourier transform limit corresponding to (a) and (c) are shown in (b) and (d) respectively.

When the energy of incident pulse was $0.8 \mathrm{~mJ}$, the pulse further reduced to $17 \mathrm{fs}$, with a Fourier transform limit of $5.5 \mathrm{fs}$. As the energy of input pulse was increased to $1.3 \mathrm{~mJ}$, filament in the tube split to multifilaments at $5 \mathrm{~cm}$ after focal point, and the spots split and converged rapidly evidenced by far field observation. The transform of multifilaments to single filament can be controlled by temperature. We could observe remarkable multifilaments with pulse energy up to $1.7 \mathrm{~mJ}$. When the temperature of heat center reached 
$170^{\circ} \mathrm{C}$, multifilaments converged to single filament and at this temperature, multifilaments will reoccur if pulse energy increases to $2.7 \mathrm{~mJ}$. When the temperature of heat center was $400^{\circ} \mathrm{C}$, multifilaments shrunk to single filament again. We increased the temperature to $450^{\circ} \mathrm{C}$, filamentation was not obvious and the pulse is self compressed to $19 \mathrm{fs}$ with a Fourier transform limit of 14.5 fs (see Fig. 19). Filament disappeared as the temperature increases to $500{ }^{\circ} \mathrm{C}$. The width of output pulse reduced to $24.5 \mathrm{fs}$ and its transform limited pulse is $15 \mathrm{fs}$ (see Fig. 19). The energy of the self compressed pusle increased by nearly $2 \mathrm{~mJ}$ compared to the case of $0.8 \mathrm{~mJ}$ (Fig. 19).

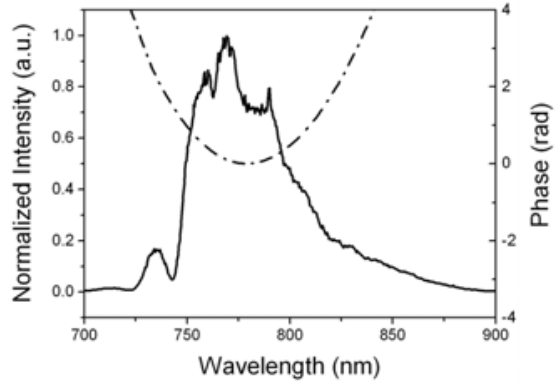

(a)

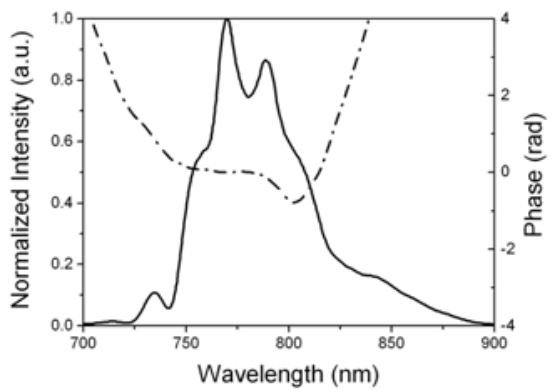

(c)

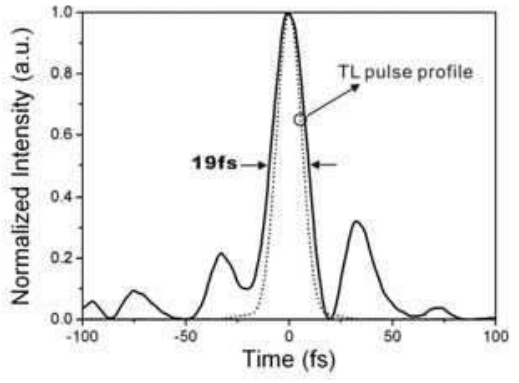

(b)

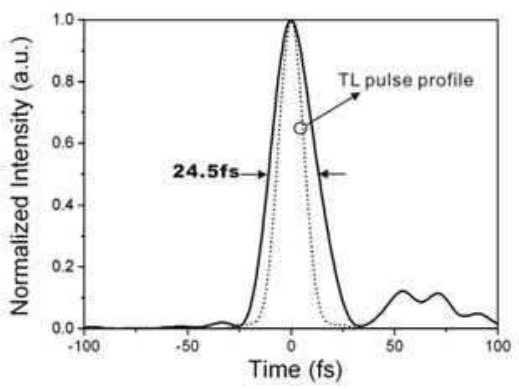

(d)

Fig. 19. The spectra and phases of (a) $450{ }^{\circ} \mathrm{C}$, (c) $500^{\circ} \mathrm{C}$ with $2.7 \mathrm{~mJ}$ incident pulse. Pulse profiles after self compression and Fourier transform limit corresponding to (a) and (c) are shown in (b) and (d) respectively.

Single filament reoccured when the energy of incident pulse increased to $5.7 \mathrm{~mJ}$, and the width of output pulse was 69 fs. Compared with multifilaments before heating, the pulse energy of self compression was increased nearly $4 \mathrm{~mJ}$ by heating the gas to $500{ }^{\circ} \mathrm{C}$ with overcoming the emergence of multifilaments.

We can define the self-compression ratio, $S$, as the ratio between the width of Fourier transform limit and that of self compression pulse which exhibits the condition of self compression without any dispersion compensation. $S=1$ is the ideal value (see table 1). Preliminary analysis shows that at the same temperature, high energy promotes self compression, but the self compression rate for high energy is low, which can approach the 
ideal value after dispersion compensation. For the same energy, self compression rate differs slightly at different gradient temperature, which is higher at lower temperature.

\begin{tabular}{l|c|c|c|c}
\hline Temperature $\left({ }^{\circ} \mathrm{C}\right)$ & $\begin{array}{c}\text { Pulse Energy } \\
(\mathrm{mJ})\end{array}$ & $\begin{array}{c}\text { Measured pulse } \\
\text { width }(\mathrm{fs})\end{array}$ & $\begin{array}{c}\text { Transform } \\
\text { limited pulse } \\
\text { width }(\mathrm{fs})\end{array}$ & $\mathrm{S}$ \\
\hline 25 (No Gradient) & 0.7 & 23.5 & 16 & 0.6809 \\
\hline 25 (No Gradient) & 0.8 & 17 & 5.5 & 0.3235 \\
\hline 450 (Gradient) & 2.7 & 19 & 14.5 & 0.7632 \\
\hline 500 (Gradient) & 2.7 & 24.5 & 15 & 0.6122 \\
\hline
\end{tabular}

Table 1 . Self compression rate $S$ at different energy and temperatre schemes.

\subsection{Pulse compression with dispersion compensation by chirp mirror after temperature controlled filamentation}

To obtain intense ultrashort pulse, dispersion compensation is need after the filamentation. We started from a $2.4 \mathrm{~mJ}$ incident pulse under $25^{\circ} \mathrm{C}$ and $2 \mathrm{~atm}$ condition, and we observed multifilaments. The width of spectrum was broaden 3 times of that of the incident pulse, and the transform limited pulse was 6 fs. The pulse compression was difficult under this condition because of the strong fluctuation caused by multifilaments. When we increased the temperature to $380{ }^{\circ} \mathrm{C}$, the pressure in the tube was about $2.3 \mathrm{~atm}$, and multifilaments gradually shrunk to a $35 \mathrm{~cm}$ long single filament, starting at $3 \mathrm{~cm}$ before the focal point. Figure 20 shows the narrowing of the pulse width due to high temperature.

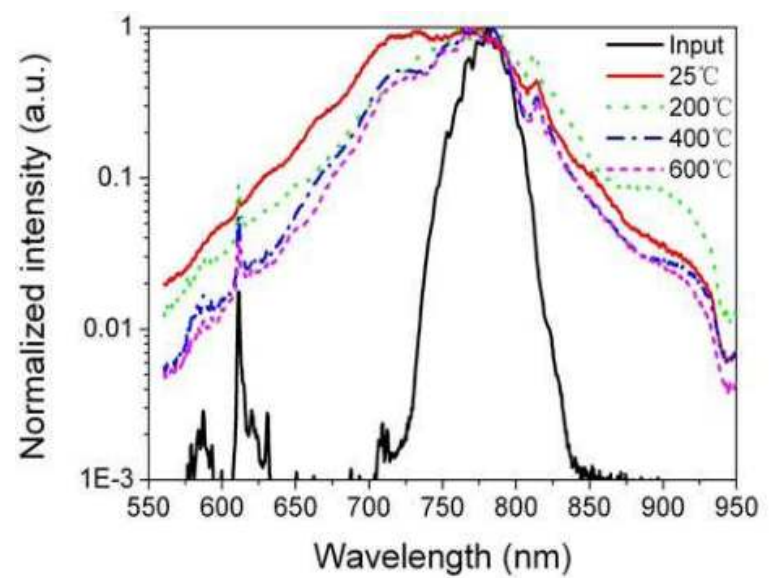

Fig. 20. Spectra of a $2.4 \mathrm{~mJ}$ incident pulse at $2 \mathrm{~atm}$ at different temperature.

In Fig. 21, we show the phases and spectrum of the pulse reflected by the chirp mirror for 6 and 8 bounces, measured by a SPIDER. We can see that after 3 bounces between the chirp mirror, the spectrum is not flat. While after 4 bounces, the spectrum becomes flat and the GDD turns from positive to negative. We can get a $1.6 \mathrm{~mJ}, 15 \mathrm{fs}$ output pulse compared with $8 \mathrm{fs}$ of transform limited,. Certainly the dispersion compensation is not complete and finely compensation is needed. 


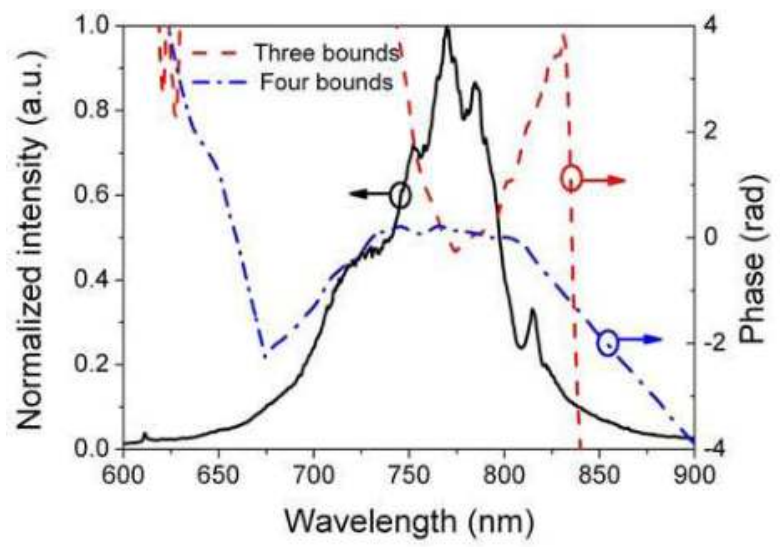

Fig. 21. Spectrum and phases for 3 and 4 bounces between chirp mirror.

\section{Summary}

In this chapter, a novel technology for generating intense few to monocycle light pulse was proposed and demonstrated. This technology has similar effect as the gradient pressure scheme while avoid the disadvantage of gas flow and consumption of expensive noble gases.

A model for simulation of the pulse evolution in a gradient temperature hollow fiber filled with argon gas has been established. The simulation results show that in the gradient temperature scheme, the incident pulse energy can be much higher than that of the uniform case, which is similar to the gradient pressure. In the gas of gradient temperature, the pulse spectra can be broadened more than that in the case of uniform temperature. Shorter pulses can be obtained after a further compression.

We also verified the effectiveness and feasibility of the scheme of gradient temperature. The entrance of the filament was heated by a furnace and the two ends of the tube were cooled with air, which resulted in the temperature gradient distribution along the tube. The presented method is easily done with only a furnace, without the large consumption of noble gas and turbulence. Although the temperature gradient is not linear, we observed that multiple filaments were shrunken into a single filament and then filament disappears by increasing the temperature to some degree, which indicates that the critical power increases with temperature due to the gas atoms squeezed to the other end of the tube where the temperature is lower. Also, the filament can appear and disappear by controlling the local temperature and incident pulse energy in turn. The spectrum of the exit pulses is not expanded so much in comparison with the case of the same pressure and the same pulse energy, because the total gas atoms number is unchanged in the sealed tube. However, higher pulse energy is allowed to incident into the tube and a round trip pass of the tube is expected to expand the spectrum further with self-compression.

The gradient temperature technique has a great advantage that the temperature is easier to control than gradient pressure by differential pumping. Another merit is that the gas in the tube is relatively steady without flow, which is very important for keeping the output spectra stable. Not long after heating the gas to a high temperature at part of the sealed tube, 
the inner gas pressure will reach an equilibrium and the gas density in the tube will be gradient while the pressure in the tube will be equal everywhere. Because the pressure in the sealed tube is uniform, the convection and instabilities does not appear in our experiments. In contrast, in our experiment, the spectra and the light spot are very stable. For the pulse of same incident peak power, the spectra expansion in the gradient temperature is not as large as in the uniform temperature case. This is because the high temperature reduces the nonlinearity. However, because of this, a higher input energy can be sent through the tube, such that at the end of the tube, the peak power of the pulse is still high enough to expand the spectrum. This is the main reason that the transform limited pulse is shorter in gradient temperature tube than in the uniform temperature one. The drawback of the scheme is that the gas density difference cannot be as large as in the scheme using differential pumping. In addition, a big temperature difference may break the glass tube.

This technique offers one more degree of freedom to control the filamentation in a gas-filled tube for the intense monocycle pulse generation without gas consumption and turbulence and opens a new way for multi $\mathrm{mJ}$ level monocycle pulse generation through filamentation in the noble gas.

\section{References}

Abramowitz, M. \& Stegun, I.A. (1972). Handbook of Mathematical Functions, Dover, ISBN : 0486-61272-4, New York

Agrawal, G.P. (2007). Nonlinear Fiber Optics (4th Edition), Academic, ISBN : 0-123-69516-3, San Diego

Akturk, S.; D’Amico, C. ; Franco, M. ; Couairon, A. \& Mysyrowicz, A. (2007). Pulse shortening, spatial mode cleaning, and intense terahertz generation by filamentation in xenon. Phys. Rev. A, Vol. 76, No. 6, (Dec. 2007) page numbers (3819-3825), ISSN: 1050-2947

Ammosov, M. V. ; Delone, N. B. \& Krainov, V. P. (1986). Tunnel ionization of complex atoms and of atomic ions in an alternating electromagnetic field. Sov. Phys. JETP, Vol. 64, No. 6, (Dec. 1986) page numbers (1191-1194), ISSN : 0038-5646

Bergé, L.; Skupin, S. ; Lederer, F. ; Méjean, G. ; Yu, J. ; Kasparian, J. ; Salmon, E. ; Wolf, J.P. ; Rodriguez, M. ; Wöste, L.; Bourayou, R. \& Sauerbrey, R. (2004). Multiple filamentation of terawatt laser pulses in air. Phys. Rev. Lett., Vol. 92, No. 22, (Jun. 2004) page numbers (225002:1-4), ISSN: 0031-9007

Braun, A.; Korn, G. ; Liu, X. ; Du, D. ; Squier J. \& Mourou, G. (1995). Self-channeling of highpeak-power femtosecond laser pulses in air. Opt. Lett., Vol. 20, No. 1, (Jan. 1995) page numbers (73-75), ISSN: 0146-9592

Cao, S.; Kong, W. ; Wang, Z. ; Song, Z. ; Qin, Y. ; Li, R. ; Wang, Q. \& Zhang Z. (2009). Filamentation control in the temperature gradient argon gas. Appl. Phys. B, Vol. 94, No. 2, (Feb. 2009) page numbers (265-271), ISSN: 0946-2171

Cook, K.; Kar, A. \& Lamb, R.A. (2005). White-light filaments induced by diffraction effects. Opt. Express, Vol. 13, No. 6, (Mar. 2005) page numbers (2025-2031), ISSN: 1094-4087

Couairon, A.; Biegert, J. ; Hauri, C.P. ; Kornelis, W.; Helbing, F.W. ; Keller, U. \& Mysyrowicz, A. (2006). Self-compression of ultra-short laser pulses down to one optical cycle by filamentation. J. Mod. Opt., Vol. 53, No. 1, (Oct. 2006) page numbers (75-85), ISSN: 0950-0340 
Couairon, A.; Franco, M. ; Mysyrowicz, A. ; Biegert, J. \& Keller, U. (2005). Pulse selfcompression to the single-cycle limit by filamentation in a gas with a pressure gradient. Opt. Lett., Vol. 30, No. 19, (Oct. 2005) page numbers (2657-2659), ISSN: 0146-9592

Courtois, C.; Couairon, A. ; Cros, B. ; Marquès, J.R. \& Matthieussent, G. (2001). Propagation of intense ultrashort laser pulses in a plasma filled capillary tube: Simulations and experiments. Phys. Plasmas, Vol. 8, No. 7, (Jul. 2001) page numbers (3445-3456), ISSN: 1070-664X

Dalgarno, A \& Kingston, A.E. (1960). The refractive indices and Verdet constants of the inert gases. Proc. R. Soc. London, Ser. A, Vol. 259, No. 1298 (Dec. 29, 1960), page numbers (424-431), ISSN : 1471-2946

Dreiskemper, R. \& Botticher, W. (1995). Current filamentation of strongly preionized high pressure glowdischarges in $\mathrm{Ne} / \mathrm{Xe} / \mathrm{HCl}$ mixtures. IEEE Transactions on Plasma Science, Vol. 23, No. 6, (Dec. 1995) page numbers (987-995), ISSN: 0093-3813

Dubietis, A.; Tamosauskas, G. ; Fibich, G. \& Ilan, B. (2004). Multiple filamentation induced by input-beam ellipticity. Opt. Lett., Vol. 29, No. 10, (May. 2004) page numbers (1126-1128), ISSN: 0146-9592

Fibich, G.; Eisenmann, S. ; Ilan, B. \& Zigler, A. (2004). Control of multiple filamentation in air. Opt. Lett., Vol. 29, No. 15, (Aug. 2004) page numbers (1772-1774), ISSN: 01469592

Fuji, T.; Horio, T. \& Suzuki, T. (2007). Generation of 12 fs deep-ultraviolet pulses by fourwave mixing through filamentation in neon gas. Opt. Lett., Vol. 32, No. 17, (Sep. 2007) page numbers (2481-2483), ISSN: 0146-9592

Hauri, C.P.; Guandalini, A.; Eckle, P.; Kornelis, W.; Biegert, J. \& Keller, U. (2005). Generation of intense few-cycle laser pulses through filamentation - parameter dependence. Opt. Express, Vol. 13, No. 19, (Sep. 2005) page numbers (7541-7547), ISSN: 1094-4087

Hauri, C. P.; Kornelis, W.; Helbing, F.W.; Heinrich, A.; Couairon, A.; Mysyrowicz, A.; Biegert, J. \& Keller, U. (2004). Generation of intense, carrier-envelope phase-locked few-cycle laser pulses through filamentation. Appl. Phys. B, Vol. 79, No. 6, (Oct. 2004) page numbers (673-677), ISSN: 0946-2171

Hosseini, S.A.; Luo, Q. ; Ferland, B. ; Liu, W. ; Chin, S.L. ; Kosareva, O.G. ; Panov, N.A. ; Aközbek, N. \& Kandidov, V.P. (2004). Competition of multiple filaments during the propagation of intense femtosecond laser pulses. Phys. Rev. A, Vol. 70, No. 03, (Sep. 2004) page numbers (3802-3813), ISSN: 1050-2947

Kandidov, V.P.; Akozbek, N. ; Scalora, M. ; Kosareva, O.G. ; Nyakk, A.V. ; Luo, Q. ; Hosseini, S.A. \& Chin, S.L. (2005). Towards a control of multiple filamentation by spatial regularization of a high-power femtosecond laser pulse. Appl. Phys. B, Vol. 80, No. 2, (Feb. 2005) page numbers (267-275), ISSN: 0946-2171

Keldysh, L.V. (1965). Ionization in the field of a strong electromagnetic wave. Sov. Phys. JETP, Vol. 20, No. 5, (May 1965) page numbers (1307-1314), ISSN : 0038-5646

Lehmeier, H.J.; Leupacher, W. \& Penzkofer, A. (1985). Nonresonant third order hyperpolarizability of rare gases and N2 determined by third harmonic generation. Opt. Commun., Vol. 56, No. 1, (Nov. 1985) page numbers (67-72), ISSN: 0030-4018

Marburger, J.H. (1975). Self-focusing: Theory. Prog. Quant. Electr. Vol. 4, page numbers (35110), ISSN: 0079-6727 
Marcatili, E.A.J. \& Schmeltzer, R.A. (1964). Hollow metallic and dielectric waveguides for long distance optical transmission and lasers (Long distance optical transmission in hollow dielectric and metal circular waveguides, examining normal mode propagation). Bell Syst. Tech. J, Vol. 43, (Jul. 1964) page numbers (1783-1809), ISSN: 0005-8580

Matsubara, E.; Yamane, K.; Sekikawa, T. \& Yamashita, M. (2007). Generation of 2.6 fs optical pulses using induced-phase modulation in a gas-filled hollow fiber. J. Opt. Soc. Am. $B$, Vol. 24, No. 4, (Apr. 2007) page numbers (985-989), ISSN: 0740-3224

Mlejnek, M.; Wright, E.M. \& Moloney, J.V. (1998). Femtosecond pulse propagation in argon: A pressure dependence study. Phys. Rev. E, Vol. 58, No. 4, (Oct. 1998) page numbers (4903-4910), ISSN: 1539-3755

Perelemov, A.M.; Popov, V.S. \&. Terent'ev, M.V. (1966). Ionization of atoms in an alternating electric field. Sov. Phys. JETP, Vol. 23, No. 5, (May 1966) page numbers (924-933), ISSN : 0038-5646

Pfeifer, T.; Gallmann, L. ; Abel, M.J. ; Neumark, D.M. \& Leone, S.R. (2006). Circular phase mask for control and stabilization of single optical filaments. Opt. Lett., Vol. 31, No. 15, (Aug. 2006) page numbers (2326-2328), ISSN: 0146-9592

Shen Y.R. (1984). The Principles of Nonlinear Optics, Wiley-Interscience, ISBN : 0-471-43080-3, NewYork

Skupin, S.; Bergé, L. ; Peschel, U. ; Lederer, F. ; Méjean, G. ; Yu, J. ; Kasparian, J. ; Salmon, E. ; Wolf, J.P. ; Rodriguez, M. ; Wöste, L. ; Bourayou, R. \& Sauerbrey, R. (2004). Filamentation of femtosecond light pulses in the air: Turbulent cells versus longrange clusters. Phys. Rev. E, Vol. 70, No. 4, (Oct. 2004) page numbers (6602-6616), ISSN: 1063-651X

Song, Z.; Qin, Y. ; Zhang, G. ; Cao, S. ; Pang, D. ; Chai, L. ; Wang, Q. ; Wang, Z. \& Zhang, Z. (2008). Femtosecond pulse propagation in temperature controlled gas-filled hollow fiber. Opt. Commun., Vol. 281, No. 15-16, (Aug. 2008a) page numbers (4109-4113), ISSN: 0030-4018

Song, Z.; Zhang, G. ; Cao, S. ; Pang, D. ; Chai, L. ; Wang, Q. \& Zhang Z. (2008b). Simulation of Femtosecond Pulse Propagation through Hollow Fibre Filled with Noble Gases of Gradient Temperature. Chin. Phys. Lett., Vol. 25, No. 1, (Jan. 2008) page numbers (129-132), ISSN: 0256-307X

Suda, A.; Hatayama, M. ; Nagasaka, K. \& Midorikawa, K. (2005). Generation of sub-10-fs, 5mJ-optical pulses using a hollow fiber with a pressure gradient. Appl. Phys. Lett., Vol. 86, No. 11, (Mar. 2005) page numbers (111116 :1-3), ISSN: 0003-6951

Sung, J.H.; Park, J.Y. ; Imran, T. ; Lee, Y.S. \& Nam, C.H. (2006). Generation of 0.2-TW 5.5-fs optical pulses at $1 \mathrm{kHz}$ using a differentially pumped hollow-fiber chirped-mirror compressor. Appl. Phys. B, Vol. 82, No. 1, (Jan. 2006) page numbers (5-8), ISSN: 0946-2171

Tempea, G. \& Brabec, T. (1998). Theory of self-focusing in a hollow waveguide. Opt. Lett., Vol. 23, No. 10, (May. 1998) page numbers (762-764), ISSN: 0146-9592

Vidal F. \& Johnston, T.W. (1996). Electromagnetic beam breakup: multiple filaments, single beam equilibria, and radiation. Phys. Rev. Lett., Vol. 77, No. 7, (Aug. 1996) page numbers (1282-1285), ISSN: 0031-9007 
Yamane, K.; Zhang, Z.; Oka, K.; Morita, R.; Yamashita, M. \& Suguro, A. (2003). Optical pulse compression to $3.4 \mathrm{fs}$ in the monocycle region by feedback phase compensation. Opt. Lett., Vol. 28, No. 22, (Nov. 2003) page numbers (2258-2260), ISSN: 0146-9592

Yamashita, M.; Yamane, K. \& Morita, R. (2006). Quasi-automatic phase-control technique for chirp compensation of pulses with over-one-octave bandwidth-generation of fewto mono-cycle optical pulses. IEEE J. Sel. Top. Quantum Electron, Vol. 12, Issue. 2, (March-April 2006) page numbers (213-222), ISSN: 1077-260X

Zaïr, A.; Guandalini, A.; Schapper, F.; Holler, M.; Biegert, J.; Gallmann, L.; Couairon, A.; Franco, M.; Mysyrowicz, A. \& Keller, U. (2007). Spatio-temporal characterization of few-cycle pulses obtained by filamentation. Opt. Express, Vol. 15, No. 9, (Apr. 2007) page numbers (5394-5404), ISSN: 1094-4087 


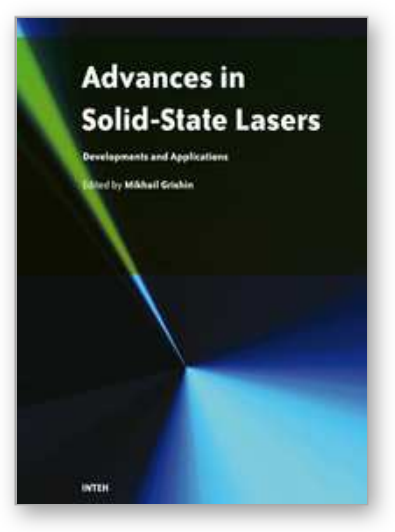

\author{
Advances in Solid State Lasers Development and Applications \\ Edited by Mikhail Grishin
}

ISBN 978-953-7619-80-0

Hard cover, 630 pages

Publisher InTech

Published online 01, February, 2010

Published in print edition February, 2010

Invention of the solid-state laser has initiated the beginning of the laser era. Performance of solid-state lasers improved amazingly during five decades. Nowadays, solid-state lasers remain one of the most rapidly developing branches of laser science and become an increasingly important tool for modern technology. This book represents a selection of chapters exhibiting various investigation directions in the field of solid-state lasers and the cutting edge of related applications. The materials are contributed by leading researchers and each chapter represents a comprehensive study reflecting advances in modern laser physics. Considered topics are intended to meet the needs of both specialists in laser system design and those who use laser techniques in fundamental science and applied research. This book is the result of efforts of experts from different countries. I would like to acknowledge the authors for their contribution to the book. I also wish to acknowledge Vedran Kordic for indispensable technical assistance in the book preparation and publishing.

\title{
How to reference
}

In order to correctly reference this scholarly work, feel free to copy and paste the following:

Zhenming Song, Yun Wei, Shiying Cao, Weipeng Kong, Dongqing Pang, Ruxin Li, Qingyue Wang and Zhigang Zhang (2010). Femtosecond Filamentation in Temperature Controlled Noble Gas, Advances in Solid State Lasers Development and Applications, Mikhail Grishin (Ed.), ISBN: 978-953-7619-80-0, InTech, Available from: http://www.intechopen.com/books/advances-in-solid-state-lasers-development-andapplications/femtosecond-filamentation-in-temperature-controlled-noble-gas

\section{INTECH}

open science | open minds

\section{InTech Europe}

University Campus STeP Ri

Slavka Krautzeka 83/A

51000 Rijeka, Croatia

Phone: +385 (51) 770447

Fax: +385 (51) 686166

www.intechopen.com

\section{InTech China}

Unit 405, Office Block, Hotel Equatorial Shanghai

No.65, Yan An Road (West), Shanghai, 200040, China

中国上海市延安西路65号上海国际贵都大饭店办公楼 405 单元

Phone: +86-21-62489820

Fax: $+86-21-62489821$ 
(C) 2010 The Author(s). Licensee IntechOpen. This chapter is distributed under the terms of the Creative Commons Attribution-NonCommercialShareAlike-3.0 License, which permits use, distribution and reproduction for non-commercial purposes, provided the original is properly cited and derivative works building on this content are distributed under the same license. 